\title{
Avaliação postural da coluna vertebral em escolares surdos de 7-21 anos
}

\section{Postural evaluation of vertebral column in deaf school kids from 7 to 21 years old}

\author{
Graziela Arruda Reinaux de Vasconcelos ${ }^{[a]}$, Pollyana Radinnê Beserra Fernandes ${ }^{[b]}$, \\ Daniella Araújo de Oliveira ${ }^{[c]}$, Etenildo Dantas Cabral ${ }^{[\mathrm{d}]}$, Lícia Vasconcelos Carvalho da Silva ${ }^{[\mathrm{e}]}$
}

[a] Aluna do curso de Fisioterapia da Associação Caruaruense de Ensino Superior (ASCES), Caruaru, PE - Brasil, e-mail: grazi_arruda@yahoo.com.br

[b] Aluna do curso de Fisioterapia da Associação Caruaruense de Ensino Superior (ASCES), Caruaru, PE - Brasil, e-mail: polly_fisiofaape@hotmail.com

[c] Fisioterapeuta, Doutora em Neuropsiquiatria e Ciências do Comportamento, docente do curso de Fisioterapia da Associação Caruaruense de Ensino Superior (ASCES), Caruaru, PE - Brasil, e-mail: sabino_daniella@ig.com.br

[d] Cirurgião-dentista, Doutor em Saúde Bucal Coletiva, docente do curso de Fisioterapia da Associação Caruaruense de Ensino Superior (ASCES) e da Universidade Federal de Pernambuco (UFPE), Caruaru, PE - Brasil, e-mail: etenildo@gmail.com

[e] Fisioterapeuta, Mestre em Neuropsiquiatria e Ciências do Comportamento, docente do curso de Fisioterapia da Associação Caruaruense de Ensino Superior (ASCES), Caruaru, PE - Brasil, e-mail: liciavcarvalho@gmail.com

\section{Resumo}

Introdução: A infância e a adolescência são períodos em que a prevenção e o tratamento de alterações posturais decorrentes do mau hábito postural e da sobrecarga biomecânica são mais eficazes, graças à maturação osteomuscular que ocorre nessas fases. Objetivo: Detectar possíveis alterações posturais da coluna vertebral em escolares surdos de 7-21 anos no Centro de Reabilitação e Educação Especial Rotary Club Caruaru. Metodologia: Trinta de dois escolares surdos participaram de uma entrevista sobre dados referentes ao período de instalação e tipo de surdez, dados biológicos, antropométricos e de hábitos posturais, e foram submetidos a uma avaliação postural de acordo com critérios propostos pela literatura. Os dados foram analisados de modo descritivo e a associação entre as variáveis utilizou o teste Qui-Quadrado ou suas variações, por meio do programa SPSS ${ }^{\circledR}$ versão 11.5. Resultados: Os resultados mostraram que a prevalência de alterações posturais nos escolares surdos foi de $90,62 \%$ dos casos. A alteração postural mais frequente foi a hipercifose torácica, que esteve associada à dor nos escolares. Considerando a distribuição por sexo, a alteração postural do tipo escoliose mostrou-se associada ao sexo feminino. Conclusão: A elevada prevalência de alterações posturais nos escolares surdos no nosso estudo sugere que a surdez pode representar um fator 
importante no desenvolvimento de alterações posturais. Entretanto, existe a necessidade de novos estudos que investiguem uma possível relação entre a surdez e fatores biológicos e ambientais em populações maiores de escolares.

Palavras-chave: Avaliação. Postura. Perda auditiva. Escoliose.

\begin{abstract}
Introduction: Childhood and teenage are periods where prevention and treatment of postural alterations, derived from bad postural habit and mechanical overcharge are more efficient, due to osteomuscular maturation that occurs in these phases. Objective: The main goal of this study was to detect possible postural alterations of the vertebral column in deaf school kids from 7 to 21 years old, at Centro de Reabilitação e Educação Especial Rotary Club Caruaru (Rehabilitation and Educational Center Rotary Club), in Pernambuco State, Brazil. Methodology: Thirty two deaf school kids have participated of an interview about data, referring to the period of installation and kind of deafness, biological, anthropometric and postural habits data, having been submitted to a postural evaluation according to literature proposed criteria. Data were analyzed in a descriptive way and association among variable points used the Chi-Square test or their variant items through SPSS ${ }^{\mathrm{TM}}$ program version 11.5. Results: Studies have showed that prevalence of postural alterations on deaf school kids reached $90.62 \%$ of all cases. The most frequently observed postural alteration was thoracic hyperciphosis, which was associated to pain in school kids. By considering the distribution in relation to sex, postural alteration of the type scoliosis was associated to female sex. Conclusion: High prevalence of postural alterations on deaf school kids in our study suggests that deafness may represent an important factor in development of postural alterations. However, there is necessity of new studies to investigate a possible relation between deafness and biological and environmental factors in larger school kids' population.
\end{abstract}

Keywords: Assessment. Posture. Hearing loss. Scoliosis.

\title{
Introdução
}

De acordo com os dados da Organização Mundial de Saúde (OMS), existem mais de 120 milhões de pessoas no mundo com perda auditiva, sendo 8,7 milhões com idade variando de 0 a 19 anos (1). No último senso do Instituto Brasileiro de Geografia e Estatística (IBGE), em 2000, a incidência de surdez entre os homens foi maior que nas mulheres, correspondendo a 103,4 e a 92,7, respectivamente, para cada 100 mil habitantes (2).

A surdez interfere de forma decisiva na formação da linguagem e no desenvolvimento de distúrbios emocionais, cognitivos, dificuldades de aprendizagem e de adaptação social (3, 4). Segundo a literatura, parece haver uma a associação direta entre surdez e alterações vestibulares, de modo que quanto mais profunda a surdez, maior a probabilidade de lesão do receptor vestibular (5). Essas alterações favorecem mudanças no tônus muscular e no alinhamento da coluna vertebral, e são responsáveis pelo desenvolvimento de dores musculares e consequente queda no rendimento escolar em jovens (6). O senso cinestésico da postura alterada, o mau hábito postural, a sobrecarga biomecânica e o desequilíbrio muscular estão diretamente relacionados ao desenvolvimento de alterações posturais (7).

A infância e a adolescência são períodos de maior importância para o desenvolvimento músculoesquelético (8). Os hábitos incorretos nessa fase favorecem a aquisição de deformidades posturais que podem resultar em prejuízos significativos aos escolares, particularmente às estruturas que compõem a coluna vertebral (9). De acordo com Zapater (9), a mudança da postura de pé para a sentada nos estudantes aumenta em aproximadamente $35 \%$ a pressão interna sobre o núcleo do disco intervertebral. Dessa forma, as principais alterações posturais evidenciadas nesses escolares são a hipercifose torácica, hiperlordose lombar e a escoliose $(7,8)$. 
A fase escolar representa o melhor momento para o estímulo de hábitos saudáveis (9, 10). Assim, na tentativa de prevenir o desenvolvimento de alterações posturais em escolares, a fisioterapia fornece orientações que visam a evitar as posições habituais ou atividades que aumentem as curvaturas da coluna vertebral dos alunos (11). Segundo Zapater (9), a fisioterapia preventiva inclui programas de educação postural que possibilitam o conhecimento acerca dos desvios posturais e o replanejamento do ambiente, com intuito de minimizar as complicações entre os escolares.

Conforme Martelli e Traebert (8), a detecção precoce das alterações posturais em escolares diminui a probabilidade de disfunções irreversíveis na coluna vertebral na fase adulta. Dessa forma, o objetivo deste estudo foi detectar possíveis alterações posturais em escolares surdos de 7-21 anos e relacionar essas alterações às características da surdez, aos dados biológicos (idade e sexo), antropométricos (peso, IMC e altura), hábitos posturais e presença de dor nos escolares.

\section{Materiais e métodos}

Foi realizado um estudo transversal no Centro de Reabilitação e Educação Especial Rotary Club Caruaru, no município de Caruaru, PE, no primeiro semestre de 2008. Foram selecionados 42 estudantes surdos de acordo com os critérios: faixa etária de 7-21 anos, ambos os sexos, devidamente matriculados e com diagnóstico de surdez segundo exame audiométrico. Dos alunos selecionados, 32 aceitaram participar da pesquisa.

O projeto de pesquisa foi submetido e aprovado pelo Comitê de Ética e Pesquisa da Associação Caruaruense de Ensino Superior (ASCES). A autorização dos participantes foi realizada por meio do Termo de Consentimento Livre e Esclarecido, de acordo com a resolução CNS 196/96, em que os voluntários tiveram o direito a participar ou não do estudo, podendo interromper a pesquisa em qualquer momento.

Os voluntários foram entrevistados e responderam a questões que avaliam características da surdez (tipo, localização e grau da surdez), dados antropométricos (peso, IMC e altura), biológicos (idade e sexo) e hábitos posturais (Anexo C). Em seguida, foram submetidos a uma avaliação postural utilizando o simetrógrafo, segundo critérios propostos por Kendall, McCreary e Provance (11) e Politano (12). Tais critérios possibilitam a identificação de alterações posturais como hipercifose, hiperlordose e escoliose. Para a avaliação postural o escolar posicionou-se na postura ortostática em frente ao simetrógrafo, adotando as posturas de frente, lateral direita e esquerda e costas. Foram utilizados para a avaliação dos escolares: simetrógrafo, fita métrica, balança e emborrachados para marcação dos pontos anatômicos no meato auditivo externo, acrômio, sétima vértebra cervical, ângulo inferior das escápulas, glabela e manúbrio esternal (11). Posteriormente, foi realizada a medição da altura (m), do peso corporal $(\mathrm{kg}$ ) e o cálculo do índice de massa corporal (IMC), e aplicado o Teste de Adams para observação ou não de gibosidade $(12,7)$.

Os escolares usaram roupas adequadas para o exame, como short e top para o sexo feminino e short para o sexo masculino. A análise estatística realizada foi exploratória descritiva e para a associação entre as variáveis utilizou-se o teste Qui-Quadrado para tendência e, quando necessário, o Teste Exato de Fisher e o Linear, por meio do programa SPSS ${ }^{\circledR} 11.5$ for Windows.

\section{Resultados}

Alunos surdos com idade entre 7-21 anos ( $\mathrm{n}=32$ ) participaram deste estudo, distribuídos segundo o sexo masculino $(n=17)$ e feminino $(n=15)$. As alterações posturais foram observadas em 29 alunos $(90,62 \%)$, sendo a hipercifose torácica $(75 \%)$ e a hiperlordose lombar $(50 \%)$ as alterações mais observadas (Tabela 1$)$.

Quanto às características da surdez, todos os participantes apresentaram surdez congênita e bilateral. Considerando o grau de surdez e o tipo de disfunção, $75 \%$ dos escolares apresentam surdez profunda e $87,5 \%$, disacusia. 
Tabela 1 - Prevalência de alterações posturais da coluna vertebral em escolares Surdos de 7-21 anos ( $\mathrm{n}=32)$

\begin{tabular}{lcc}
\hline $\begin{array}{l}\text { Alterações posturais } \\
\text { da coluna vertebral }\end{array}$ & $\mathbf{n}$ & $\%$ \\
\hline Coluna Cervical & 31 & 96,9 \\
$\quad$ Normal & 1 & 3,1 \\
Retificada & & \\
Coluna Torácica & 8 & 25 \\
Normal & 24 & 75 \\
Hipercifose & & \\
Coluna Lombar & 13 & 40,6 \\
Normal & 16 & 50 \\
Hiperlordose & 3 & 9,4 \\
Retificada & & \\
Escoliose & 20 & 62,5 \\
Ausente & 12 & 37,5 \\
Presente & 8 & 66,7 \\
Funcional & 4 & 33,3 \\
Estrutural & & \\
\hline & & \\
\hline
\end{tabular}

A Tabela 2 mostra os resultados do teste de associação entre presença de alterações posturais e variáveis biológicas, antropométricas e presença de dor. O sexo feminino mostrou-se associado à prevalência de alteração postural do tipo escoliose $(\mathrm{p}=0,027)$, e a dor esteve associada à alteração postural do tipo hipercifose torácica $(\mathrm{p}=0,023)$. Entretanto, nenhuma relação foi estabelecida entre a presença de alteração postural e as variáveis altura, peso, IMC e hábitos posturais (Tabela 3).

Tabela 2 - Associação entre alteração postural da coluna vertebral, hipercifose torácica, hiperlordose lombar, escoliose e dados biológicos, antropométricos e de presença de dor em escolares surdos de 7-21 anos ( $\mathrm{n}=29$ )

(continua)

\begin{tabular}{lcccccccccccc}
\hline Variáveis & $\begin{array}{c}\text { Presença de } \\
\text { alteração postural da } \\
\text { coluna vertebral }\end{array}$ & \multicolumn{2}{c}{$\begin{array}{c}\text { Presença de } \\
\text { hipercifose torácica }\end{array}$} & $\begin{array}{c}\text { Presença de } \\
\text { hiperlordose lombar }\end{array}$ & Presença de escoliose \\
& $\mathbf{( \% )}$ & $\mathbf{n}$ & $\boldsymbol{p}^{*}$ & $\mathbf{( \% )}$ & $\mathbf{n}$ & $\boldsymbol{p}^{*}$ & $\mathbf{( \% )}$ & $\mathbf{n}$ & $\boldsymbol{p}^{*}$ & $\mathbf{( \% )}$ & $\mathbf{n}$ & $\boldsymbol{p}^{*}$ \\
\hline Idade (anos) & & & & & & & & & & & & \\
$<$ ou = 12 & 44,8 & 13 & & 41,7 & 10 & & 41,7 & 10 & 50,0 & 6 \\
$13-18$ & 37,9 & 11 & 0,382 & 37,5 & 9 & 0,167 & 37,5 & 9 & 0,741 & 41,7 & 5 & 0,536 \\
$>18$ & 17,2 & 5 & & 20,8 & 5 & & 20,8 & 5 & & 8,3 & 1
\end{tabular}


Tabela 2 - Associação entre alteração postural da coluna vertebral, hipercifose torácica, hiperlordose lombar, escoliose e dados biológicos, antropométricos e de presença de dor em escolares surdos de 7-21 anos (n=29)

(conclusão)

\begin{tabular}{|c|c|c|c|c|c|c|c|c|c|c|c|c|}
\hline \multirow[t]{2}{*}{ Variáveis } & \multicolumn{3}{|c|}{$\begin{array}{c}\text { Presença de } \\
\text { alteração postural da } \\
\text { coluna vertebral }\end{array}$} & \multicolumn{3}{|c|}{$\begin{array}{c}\text { Presença de } \\
\text { hipercifose torácica }\end{array}$} & \multicolumn{3}{|c|}{$\begin{array}{c}\text { Presença de } \\
\text { hiperlordose lombar }\end{array}$} & \multicolumn{3}{|c|}{ Presença de escoliose } \\
\hline & $(\%)$ & $\mathbf{n}$ & $p^{*}$ & $(\%)$ & $\mathrm{n}$ & $p^{*}$ & $(\%)$ & $\mathbf{n}$ & $p^{*}$ & $(\%)$ & $\mathrm{n}$ & $p^{*}$ \\
\hline \multicolumn{13}{|l|}{ Sexo } \\
\hline Masculino & 51,7 & 15 & \multirow{2}{*}{1,000} & 50,0 & 12 & \multirow{2}{*}{0,691} & 50,0 & 8 & \multirow{2}{*}{0,867} & 25,0 & 3 & \multirow{2}{*}{0,027} \\
\hline Feminino & 48,3 & 14 & & 50,0 & 12 & & 50,0 & 8 & & 75,0 & 9 & \\
\hline \multicolumn{13}{|l|}{$\operatorname{Altura(m)}$} \\
\hline$<\mathrm{ou}=1,50$ & 34,5 & 10 & \multirow{2}{*}{0,540} & 29,2 & 7 & \multirow{2}{*}{0,116} & 37,5 & 6 & \multirow{2}{*}{0,202} & 41,7 & 5 & \multirow{2}{*}{0,724} \\
\hline$>1,50$ & 65,5 & 19 & & 70,8 & 17 & & 62,5 & 10 & & 58,3 & 7 & \\
\hline \multicolumn{13}{|l|}{ Peso(kg) } \\
\hline$<\mathrm{ou}=45$ & 51,7 & 15 & \multirow{2}{*}{1,000} & 50,0 & 12 & \multirow{2}{*}{0,691} & 56,3 & 9 & \multirow{2}{*}{0,705} & 66,7 & 8 & \multirow{2}{*}{0,291} \\
\hline$>45$ & 48,3 & 14 & & 50,0 & 12 & & 43,8 & 7 & & 33,3 & 4 & \\
\hline \multicolumn{13}{|l|}{ IMC } \\
\hline Baixo Peso & 48,3 & 14 & \multirow{3}{*}{0,476} & 50,0 & 12 & \multirow{3}{*}{0,645} & 50,0 & 8 & \multirow{3}{*}{0,695} & 50,0 & 6 & \multirow{3}{*}{0,537} \\
\hline Normal & 41,4 & 12 & & 37,5 & 9 & & 37,5 & 6 & & 50,0 & 6 & \\
\hline Obesidade grau I & 10,3 & 3 & & 12,5 & 3 & & 12,5 & 2 & & 0,0 & 0 & \\
\hline \multicolumn{13}{|l|}{ Dor } \\
\hline Presente & 86,2 & 25 & \multirow{2}{*}{0,083} & 91,7 & 22 & \multirow{2}{*}{0,023} & 81,3 & 13 & \multirow{2}{*}{0,429} & 83,3 & 10 & \multirow{2}{*}{1,000} \\
\hline Ausente & 13,8 & 4 & & 8,3 & 2 & & 18,8 & 3 & & 16,7 & 2 & \\
\hline
\end{tabular}

*Exato de Fisher ou Linear de Associação.

Tabela 3 - Associação entre alteração postural da coluna vertebral, hipercifose torácica, hiperlordose lombar, escoliose e hábitos posturais em escolares surdos de 7-21 anos $(\mathrm{n}=29)$

(continua)

\begin{tabular}{|c|c|c|c|c|c|c|c|c|c|c|c|c|}
\hline \multirow[t]{2}{*}{ Variáveis } & \multicolumn{3}{|c|}{$\begin{array}{l}\text { Presença de } \\
\text { alteração postural da } \\
\text { coluna vertebral }\end{array}$} & \multicolumn{3}{|c|}{$\begin{array}{c}\text { Presença de } \\
\text { hipercifose torácica }\end{array}$} & \multicolumn{3}{|c|}{$\begin{array}{c}\text { Presença de } \\
\text { hiperlordose lombar }\end{array}$} & \multicolumn{3}{|c|}{ Presença de escoliose } \\
\hline & $(\%)$ & $\mathbf{n}$ & $p^{*}$ & $(\%)$ & $\mathrm{n}$ & $p^{*}$ & $(\%)$ & $\mathrm{n}$ & $p^{*}$ & $(\%)$ & $\mathrm{n}$ & $p^{*}$ \\
\hline \multicolumn{13}{|c|}{ Postura dormir } \\
\hline Bruços & 51,7 & 15 & \multirow{4}{*}{0,098} & 54,2 & 13 & \multirow{4}{*}{0,116} & 50,0 & 8 & \multirow{4}{*}{0,138} & 33,3 & 4 & \multirow{4}{*}{0,815} \\
\hline Costas & 34,5 & 10 & & 33,3 & 8 & & 31,3 & 5 & & 50,0 & 6 & \\
\hline De Lado & 6,9 & 2 & & 8,3 & 2 & & 12,5 & 2 & & 16,7 & 2 & \\
\hline Outros & 6,9 & 2 & & 4,2 & 1 & & 6,3 & 1 & & 0,0 & 0 & \\
\hline
\end{tabular}


Tabela 3 - Associação entre alteração postural da coluna vertebral, hipercifose torácica, hiperlordose lombar, escoliose e hábitos posturais em escolares surdos de 7-21 anos ( $\mathrm{n}=29)$

(conclusão)

\begin{tabular}{|c|c|c|c|c|c|c|c|c|c|c|c|c|}
\hline \multirow[t]{2}{*}{ Variáveis } & \multicolumn{3}{|c|}{$\begin{array}{c}\text { Presença de } \\
\text { alteração postural da } \\
\text { coluna vertebral }\end{array}$} & \multicolumn{3}{|c|}{$\begin{array}{c}\text { Presença de } \\
\text { hipercifose torácica }\end{array}$} & \multicolumn{3}{|c|}{$\begin{array}{c}\text { Presença de } \\
\text { hiperlordose lombar }\end{array}$} & \multicolumn{3}{|c|}{ Presença de escoliose } \\
\hline & $(\%)$ & n & $p^{*}$ & $(\%)$ & $\mathrm{n}$ & $p^{*}$ & $(\%)$ & $\mathrm{n}$ & $p^{*}$ & $(\%)$ & $\mathrm{n}$ & $p^{*}$ \\
\hline \multicolumn{13}{|l|}{ Local ver TV } \\
\hline Chão & 20,7 & 6 & & 20,8 & 5 & & 12,5 & 2 & & 25,0 & 3 & \\
\hline Cama & 37,9 & 11 & 0,090 & 41,7 & 10 & 0,146 & 43,8 & 7 & 0,668 & 25,0 & 3 & 0,859 \\
\hline Sofá & 41,4 & 12 & & 37,5 & 9 & & 43,8 & 7 & & 50,0 & 6 & \\
\hline \multicolumn{13}{|l|}{$\begin{array}{l}\text { Transporte } \\
\text { material escolar }\end{array}$} \\
\hline Mochila & 20,7 & 6 & & 25,0 & 6 & & 12,5 & 2 & & 16,7 & 2 & \\
\hline Mãos & 44,8 & 13 & 0,748 & 41,7 & 10 & 0,564 & 50,0 & 8 & 0,200 & 50,0 & 6 & 0,796 \\
\hline Bolsa universitária & 34,5 & 10 & & 33,3 & 8 & & 37,5 & 6 & & 33,3 & 4 & \\
\hline \multicolumn{13}{|l|}{$\begin{array}{l}\text { Realiza tarefa } \\
\text { escolar }\end{array}$} \\
\hline No chão & 10,3 & 3 & \multirow{4}{*}{0,596} & 12,5 & 3 & \multirow{4}{*}{0,892} & 0,0 & 0 & \multirow{4}{*}{0,120} & 16,7 & 2 & \multirow{4}{*}{0,855} \\
\hline $\mathrm{Na}$ mesa & 65,5 & 19 & & 66,7 & 16 & & 62,5 & 10 & & 58,3 & 7 & \\
\hline Na cama & 13,8 & 4 & & 8,3 & 2 & & 18,8 & 3 & & 8,3 & 1 & \\
\hline No sofá & 10,3 & 3 & & 12,5 & 3 & & 18,8 & 3 & & 16,7 & 2 & \\
\hline \multicolumn{13}{|l|}{ Dominância } \\
\hline Destro & 86,2 & 25 & \multirow{2}{*}{1,000} & 87,5 & 21 & \multirow{2}{*}{1,000} & 87,5 & 14 & \multirow{2}{*}{0,300} & 83,3 & 10 & \multirow{2}{*}{0,620} \\
\hline Sinistro & 13,8 & 4 & & 12,5 & 3 & & 12,5 & 2 & & 16,7 & 2 & \\
\hline
\end{tabular}

*Exato de Fisher ou Linear de Associação.

\section{Discussão}

No presente estudo, a prevalência de alterações posturais em escolares surdos foi de $90,62 \%$ dos casos, corroborando com os achados de Detsch (13) em escolares de 14 a 18 anos, e de Detsch e Candotti (14), em meninas de 6 a 17 anos sem deficiência auditiva, que observaram alterações posturais em 70,78\% da amostra. Porém, foi considerada alta quando comparada ao estudo de Martelli e Traebert (8) em escolares de 10 a 16 anos de idade não surdos, cuja observação revelou que $28,2 \%$ dos escolares apresentavam alterações posturais. A diferença nesses resultados talvez esteja relacionada à caracterização da amostra e ao número de alunos avaliados. No nosso estudo, a amostra foi composta por apenas 32 escolares, de ambos os sexos, apresentando surdez congênita.

A hipercifose torácica foi a alteração postural mais prevalente neste estudo (75\%), ao contrário do observado por Detsch e Candotti (14), em que apenas 10,39\% dos escolares não surdos de 6 a 17 anos apresentavam esse tipo de alteração. Kussuki, João, Cunha (15) e Penha et al. (16) relataram que o desenvolvimento de alterações posturais da coluna vertebral pode estar associado às alterações fisiológicas naturais do 
crescimento e do desenvolvimento da criança. Conforme Rosa Neto (17), nas crianças em desenvolvimento as alterações posturais decorrem não somente das atividades diárias, mas também do desequilíbrio postural gerado pelas novas proporções corporais.

No presente estudo, a hiperlordose lombar foi observada em 50\% da amostra. Por outro lado, Detsch e Candotti (14) e Martelli e Traebert (8) afirmaram que a hiperlordose lombar é a alteração postural mais prevalente em escolares sem deficiência auditiva. Segundo Brighetti e Bankoff (18) e Clark (19) a hiperlordose lombar corresponde a uma compensação da hipercifose torácica ou vice-versa. Considerando que neste estudo a hipercifose torácica foi a alteração mais frequente, essa informação poderia justificar a observação da hiperlordose lombar como a segunda alteração mais prevalente. Entretanto, não encontramos dados na literatura referente às alterações posturais mais frequentes em surdos que nos permitissem fazer uma correlação.

Durante o desenvolvimento da criança, o abdome torna-se mais efetivo principalmente entre $10 \mathrm{e}$ 12 anos de idade, o que explicaria uma redução da hiperlordose lombar no decorrer da idade devido a uma diminuição do abdome protruso (16). Entretanto, neste estudo não observamos associação entre a presença de alterações posturais e a idade do escolar surdo.

A escoliose foi a terceira alteração postural mais observada $(37,5 \%)$ nos escolares surdos, sendo mais frequente no sexo feminino, corroborando com os estudos de Motta (20) e Correa, Pereira e Silva (21) em escolares sem deficiência auditiva. As novas proporções fisiológicas adquiridas na fase da puberdade poderiam predispor as meninas a apresentar aumento das curvas da coluna além dos 10 graus $(21,22)$. Correa, Pereira e Silva (21) compreendem que o período ideal para triagem de avaliação em meninas deve preceder a fase de estirão do crescimento, próximo dos 9 até os 11 anos de idade. Mirtz (23) relatou que, dentre as alterações posturais em idade escolar, a escoliose foi o principal problema ortopédico, e associou esse achado a fatores ergonômicos da escola.

Em estudantes com deficiência auditiva, Woods (24) detectou a incidência de 1,2\% de escoliose idiopática. Esse autor sugeriu que a disfunção vestibular presente nos surdos é contornada pelo uso de outros sistemas sensoriais, reduzindo a predisposição para a escoliose nos deficientes auditivos. Grivas (22) e Motta (20), em seus estudos com escolares sem deficiência auditiva, revelaram que as alterações posturais do tipo escoliose funcional decorrem do mau posicionamento na postura sentada ou dos hábitos unilaterais no transporte dos materiais escolares (25).

Considerando a forma de transportar material escolar, não foi observada associação com a presença de alteração postural nos escolares. Em alunos sem deficiência auditiva, Kussuki, João, Cunha (15) e Brighetti e Bankoff (18) afirmaram que alguns desvios podem estar sendo provocados pela forma inadequada de carregar mochilas (peso excessivo), predispondo à hipercifose postural, em virtude da flexão de tronco. Rebelatto, Caldas e Vitta (26) relataram que os pesos médios transportados pelos escolares não surdos do sexo masculino é superior ao carregado pelo sexo feminino. Entretanto, Grimmer (27) afirmou que o peso da mochila escolar não interfere de forma direta nas alterações posturais ântero-posteriores, já que o deslocamento do centro de gravidade que ocorre diante do excesso de peso se dá de forma linear ao longo da coluna vertebral. Reforçamos, porém, a carência na literatura de pesquisas que relacionam hábitos posturais e escolares surdos.

Considerando as posturas adotadas para dormir, o maior número de alterações posturais foi observado nos escolares que adotam o decúbito dorsal ou a postura de bruços. Porém, segundo Hawes e O'Brien (28) e Kendall, McCreary e Provance (11), crianças tendem a desenvolver desequilíbrios musculares e possíveis alterações posturais quando adotam posturas assimétricas repetitivas, principalmente quando se torna um hábito curvar-se para o mesmo lado em diferentes posições posturais.

Analisando os hábitos posturais adotados para ver TV, no presente estudo não foi observada associação com presença de alteração postural. Porém, a literatura afirma que o tipo de colchão e sua maciez podem permitir um mau alinhamento postural (11), favorecendo a instalação de alterações posturais.

Avaliando a dominância manual nos escolares com deficiência auditiva, não observamos associação com presença de alteração postural. Alguns autores relatam que a escoliose é mais comum no sexo feminino, com predomínio para o lado direito $(19,11)$. Segundo Clark (19) e Kendall, McCreary e Provance (11), a 
população destra está mais suscetível ao desenvolvimento de escoliose do que os sinistros, que possuem menores alterações posturais porque precisam executar suas atividades nas cadeiras que são elaboradas para o uso da mão direita.

A dor esteve presente em 96,2\% dos estudantes surdos com alteração postural, revelando associação com a presença de hipercifose torácica. Segundo Kendall, McCreary e Provance (11), a dor surge a partir do acúmulo de sobrecargas biomecânicas repetidas por um período longo. Detsch (13) relatou que muitas posturas corporais adotadas no dia a dia interferem nas estruturas anatômicas, aumentando o estresse sobre a coluna vertebral e provocando desconfortos, dores ou incapacidades funcionais. Além disso, a posição de hipercifose torácica encontrada em crianças em idade escolar pode estar associada a alterações ergonômicas das cadeiras escolares e causas comportamentais, como a tentativa das meninas em esconder o desenvolvimento dos seios $(16,14)$.

Embora não tenha existido uma associação estatística entre as variáveis idade e presença de alteração postural, observou-se que os escolares mais velhos apresentaram maior frequência de alterações posturais que os mais jovens. Por causa da insuficiência de trabalhos publicados relacionando os surdos com variáveis biológicas, não foi possível justificar esses achados. Entretanto, Detsch e Candotti (14), em seu estudo com escolares não surdos, demonstraram também maiores resultados de alterações posturais em escolares mais velhos. Por outro lado, no estudo de Detsch (13) as alterações posturais não revelaram associação com a idade, porém, seus dados discordam do presente estudo sugerindo que as alterações posturais tendem a diminuir com o avançar da idade. Os dados divergem também de Penha et al. (16) e Kendall, McCreary e Provance (11), que afirmaram que a maior flexibilidade na infância favorece o desenvolvimento das alterações na postura, enquanto em adultos esses desvios são menos evidentes.

Detsch (13) relatou que estudantes com sobrepeso ou obesidade apresentaram maior prevalência de alterações posturais quando comparados aos estudantes com IMC normal, justificando esse achado pela alteração no centro de gravidade por excesso de massa corporal presente nos estudantes com sobrepeso ou obesidade. Taylor (29) ressaltou que em crianças com sobrepeso a probabilidade de dores musculoesqueléticas e de fraturas é maior graças ao comprometimento da mobilidade. Kussuki, João e Cunha (15) salientaram que a depressão é comum em crianças obesas e que estas passam a adotar uma postura introvertida, que também pode contribuir para a instalação de alterações posturais.

No presente estudo, as alterações posturais foram observadas nos escolares, independente dos valores de peso, IMC e altura. No estudo de Martelli e Traebert (8) em alunos sem deficiência auditiva, a altura revelou associação com alterações posturais; assim, escolares mais baixos apresentaram maiores alterações posturais que os mais altos. Segundo esses autores, pode haver diferenças de prevalência de alteração postural entre os escolares de mesmo sexo, idade, altura e peso, em decorrência de diferenças biológicas nos padrões de crescimento e desenvolvimento. Entretanto, não há pesquisas com surdos que permitam comparação entre os dados.

Neste contexto, ressaltamos a necessidade de programas direcionados à saúde escolar que reforcem a prática de exercícios físicos e de manutenção da boa postura, além do incentivo ao treinamento de professores para que forneçam informações sobre situações de risco do ambiente escolar. O conhecimento de alterações posturais encontradas também em crianças portadoras de deficiência auditiva reforça a importância da implementação do fisioterapeuta na escola.

\section{Considerações finais}

A elevada prevalência de alterações posturais nos escolares surdos neste estudo não permitiu a associação entre essa variável e fatores biológicos, antropométricos e ambientais, sugerindo que a surdez pode representar um fator importante no desenvolvimento dessas alterações nos escolares.

Reconhecemos que o tamanho da amostra pode ter sido um fator limitante deste estudo; assim, os nossos achados não podem representar dados conclusivos. Por outro lado, a insuficiência de trabalhos publicados sobre a avaliação postural de indivíduos surdos reforça a necessidade de novos estudos. 


\section{Referências}

1. Silva EJC, Llerena JJC, Cardoso MHCA. Estudo seccional descritivo de crianças com deficiência auditiva atendidas no Instituto Nacional de Educação de Surdos, Rio de Janeiro, Brasil. Cad Saúde Pública. 2007;23(3):627-36.

2. Instituto Brasileiro de Geografia e Estatística - IBGE. As mulheres no alvo das políticas públicas brasileiras. [bomepage na internet], [acesso em 24 ago. 2008]. Disponível em: http://www.ibge.gov.br/home/presidencia/ noticias $/$ noticia_visualiza.php?id_noticia $=605 \&$ id_pagina $=1$

3. Cecatto SB, Garcia RID, Costa KS, Abdo TRT, Rezende CEB, Rapoport PB. Análise das principais etiologias de deficiência auditiva em Escola Especial "Anne Sullivan". Rev Bras Otorrinolaringol. 2003;69(2):235-40.

4. Silva LPA, Queiros F, Lima I. Fatores etiológicos da deficiência auditiva em crianças e adolescentes de um centro de referência APADA em Salvador-BA. Rev Bras Otorrinolaringol. 2006;72(1):33-6.

5. Suarez H, Angeli S, Suarez A, Rosales B, Carrera X, Alonso R. Balance sensory organization in children with profound hearing loss and cochlear implants. Int J Pediatr Otorhinolaryngol. 2007;71(4):629-37.

6. Tribastone F. Tratado de exercícios corretivos aplicados à reeducação motora postural. São Paulo: Manole; 2001.

7. Kisner C, Colby LA. Exercícios terapêuticos: fundamentos e técnicas. 4a ed. São Paulo: Manole; 2005.

8. Martelli RC, Traebert J. Estudo descritivo das alterações posturais de coluna vertebral em escolares de 10 a 16 anos de idade: Tangará-SC, 2004. Rev Bras Epidemiol. 2006;9(1)87-93.

9. Zapater AR, Silveira DM, Vitta A, Padovani CR, Silva JCP. Postura sentada: a eficácia de um programa de educação para escolares. Ciênc Saúde Coletiva. 2004;9(1):191-9.

10. Geldhof E, Cardon G, Bourdeaudhuij I, Clercq D. Back posture education in elementary schoolchildren: a 2-year follow-up study. Eur Spine J. 2007;16(6):841-50.

11. Kendall FP, McCreary EK, Provance PG. Músculos: provas e funções. 4a ed. São Paulo: Manole; 1995.

12. Politano RC. Levantamento dos desvios posturais em adolescentes de 11 a 15 anos em escola estadual do município de Cacoal-RO [dissertação]. Brasília: Universidade de Brasília; 2006.

13. Detsch C, Luz AMH, Candotti CT, Oliveira DS, Lazaron F, Guimarães LK, et al. Prevalência de alterações posturais em escolares do ensino médio em uma cidade no Sul do Brasil. Rev Panam Salud Publica. 2007;21(4):231-8.

14. Detsch C, Candotti CT. A incidência de desvios posturais em meninas de 6 a 17 anos da cidade de Novo Hamburgo. Movimento. 2001;7(15):43-56.

15. Kussuki, MAM, João SMA, Cunha ACP. Caracterização postural da coluna de crianças obesas de 7 a 10 anos. Fisioter Mov. 2007;20(1):77-84.

16. Penha PJ, João SMA, Casarotto RA, Amino CJ, Penteado DC. Postural assessment of girls between 7 and 10 years of age. Clinics. 2005; 60(1):9-16.

17. Rosa Neto F. Avaliação postural em escolares de $1^{\text {a }}$ a $4^{a}$ série do $1^{\circ}$ grau. Rev Bras Ciênc Mov. 1991;5(2):7-11.

18. Brighetti V, Bankoff ADP. Levantamento da incidência de cifose postural e ombros caídos em alunos de $1^{\mathrm{a}}$ a $4^{\mathrm{a}}$ séries escolar. Rev Bras Ciênc Esporte. 1986;7(3):93-7.

19. Clark WA. Postural defects-correctable in school physical education classes. Cal West Med. 1928;28(5):636-9.

20. Mota JAPS. A postura como fator de observação na escola. Rev Bras Ciênc Mov. 1991;5(2):36-40.

21. Correa AL, Pereira JS, Silva MAG da. Avaliação dos desvios posturais em escolares: estudo preliminar. Fisioter Bras. 2005;6(3):175-78. 
22. Grivas TB, Wade MH, Negrini S, O'Brien JP, Maruyama T, Hawes MC, et al. SOSORT consensus paper: school screening for scoliosis. Where are we today? Scoliosis. 2007;2(17):1-23.

23. Mirtz TA, Thompson MA, Greene L, Wyatt LA, Akagi CG. Adolescent idiopathic scoliosis screening for school, community, and clinical health promotion practice utilizing the PRECEDE-PROCEED model. Chiropr Osteopat. 2005;13(25):1-11.

24. Woods LA, Haller RJ, Hansen PD, Fukumoto DE, Herman RM. Decreased incidence of scoliosis in hearingimpaired children: implications for a neurologic basis for idiopathic scoliosis. Spine. 1995;20(7):776-81.

25. Negrini S, Negrini A. Postural effects of symmetrical and asymmetrical loads on the spines of schoolchildren. Scoliosis. 2007;2(8):1-7.

26. Rebelatto JR, Caldas MAJ, Vitta A. Influência do transporte do material escolar sobre a ocorrência de desvios posturais em estudantes. Rev Bras Ortop. 1991;26(11-12):403-10.

27. Grimmer K, Dansie B, Milanese S, Pirunsan U, Trott P. Adolescent standing postural response to backpack loads: a randomised controlled experimental study. BMC Musculoskelet Disord. 2002;3(10):1-10.

28. Hawes MC, O'Brien JP. The transformation of spinal curvature into spinal deformity: pathological processes and implications for treatment. Scoliosis. 2006;1(3):1-9.

29. Taylor ED, Theim KR, Mirch MC, Ghorbani S, Tanofsky-Kraff M, Adler-Wailes DC, et al. Orthopedic complications of overweight in children and adolescents. Pediatrics. 2006;117(6):2167-74.

Recebido: $17 / 12 / 2008$

Received: $12 / 17 / 2008$

Aprovado: 06/05/2010 Approved: 05/06/2010

Revisado: 23/06/2010

Reviewed: 06/23/2010 